\title{
Effects of lysine to arginine mutations in HIV-1 Vif on its expression and viral infectivity
}

\author{
BOONRUANG KHAMSRI $^{1,2}$, MIKAKO FUJTTA ${ }^{1}$, KAZUYA KAMADA ${ }^{1}$, AHMAD PIROOZMAND $^{1}$, \\ TOMOKI YAMASHITA ${ }^{1}$, TSUNEO UCHIYAMA ${ }^{1}$ and AKIO ADACHI ${ }^{1}$ \\ ${ }^{1}$ Department of Virology, Institute of Health Biosciences, The University of Tokushima \\ Graduate School, Tokushima 770-8503, Japan; ${ }^{2}$ Department of Microbiology and Parasitology, \\ Faculty of Medical Science, Naresuan University, Phitsanulok 65000, Thailand
}

Received May 17, 2006; Accepted July 5, 2006

\begin{abstract}
We previously demonstrated that the expression in cells of human immunodeficiency virus type 1 (HIV-1) Vif is maintained at low level by proteasome-degradation. We examined the contribution of 16 lysines present in Vif (NL432 clone), which is composed of 192 amino acids (aa), to its expression within cells and to viral infectivity for nonpermissive cells. To this end, various lysine-arginine mutations were introduced into wild-type (wt) Vif, and the mutational effects were monitored by transfection experiments. When all the lysines were changed to arginines, the mutant Vif was expressed in cells at much higher level than wt and was much more stable. Both N-terminal (aa nos. 34 and 36) and C-terminal (aa nos. 179 and 181) lysines were found to be almost sufficient for wt property. Different from this observation, one of the lysines at aa nos. 22 and 26 was demonstrated to be essential for the virus to grow in nonpermissive cells. Our results showed that there is no clear corelationship between the expression level of HIV-1 Vif and viral infectivity.
\end{abstract}

\section{Introduction}

Vif is one of the human immunodeficiency virus type 1 (HIV-1) accessory proteins, and is conserved in all known primate immunodeficiency viruses (1). It is dispensable for the replication of HIV-1 in permissive cells like MT-4 (2) and M8166 (3), but is critical for the viral growth in nonpermissive cells such as H9 (4) and peripheral blood mononuclear cells (5-9). Recent studies have shown that the non-permissive cells have a cytidine deaminase APOBEC3G

Correspondence to: Dr Akio Adachi, Department of Virology, Institute of Health Biosciences, The University of Tokushima Graduate School, 3-18-15 Kuramoto-cho, Tokushima-shi, Tokushima 770-8503, Japan

E-mail: adachi@basic.med.tokushima-u.ac.jp

Key words: HIV-1, Vif, lysine, arginine carrying anti-viral activity, and that the Vif counteracts the virion incorporation of APOBEC3G (10-19). The precise molecular mechanism for this activity of Vif, however, remains to be elucidated.

We have recently demonstrated that the expression of HIV-1 Vif is controlled uniquely to be at low level among accessory proteins by proteasome degradation $(20,21)$. Virological significance of this degradation can be explained by the fact that a high expression level of Vif inhibits viral infectivity through modulating proteolytic processing of the Gag precursor at the $\mathrm{p} 2 /$ nucleocapsid processing site (22). However, the experiments were done in a quite artificial system; expression of a large amount of Vif by pNL-A1 (23) and examination of infectivity by a single-round replication assay.

In this study, we investigated the relationship between the Vif expression level within cells and viral multi-cycle infectivity for the non-permissive cells. For this purpose, we introduced a wide variety of lysine to arginine mutations into wild-type (wt) Vif, because it is well known that proteins are poly-ubiquitinated at their lysine residues or $\mathrm{N}$-terminus to become a marker recognized by the proteasome, and that the polyubiquitinated proteins are then degraded (24); the lysine and arginine have similar physicochemical characteristics. We identified lysines in Vif which are important for the wt expression level within cells and for the viral multi-cycle infectivity in non-permissive cells.

\section{Materials and methods}

Cells. A lymphocytic cell line H9 (4) was cultured in RPMI1640 medium supplemented with $10 \%$ heat-inactivated fetal bovine serum (FBS). A monolayer cell line 293T (25) was cultured in Eagle's minimal essential medium supplemented with $10 \%$ heat-inactivated FBS.

Transfection. For transfection of plasmid DNAs into adherent 293 T cells, the calcium-phosphate coprecipitation technique (26) or the Lipofectamine Plus ${ }^{\mathrm{TM}}$ system (Invitrogen, Carlsbad, CA, USA) was used. For transfection of lymphocytic H9 cells, the electroporation method was used as previously described (26). 
A

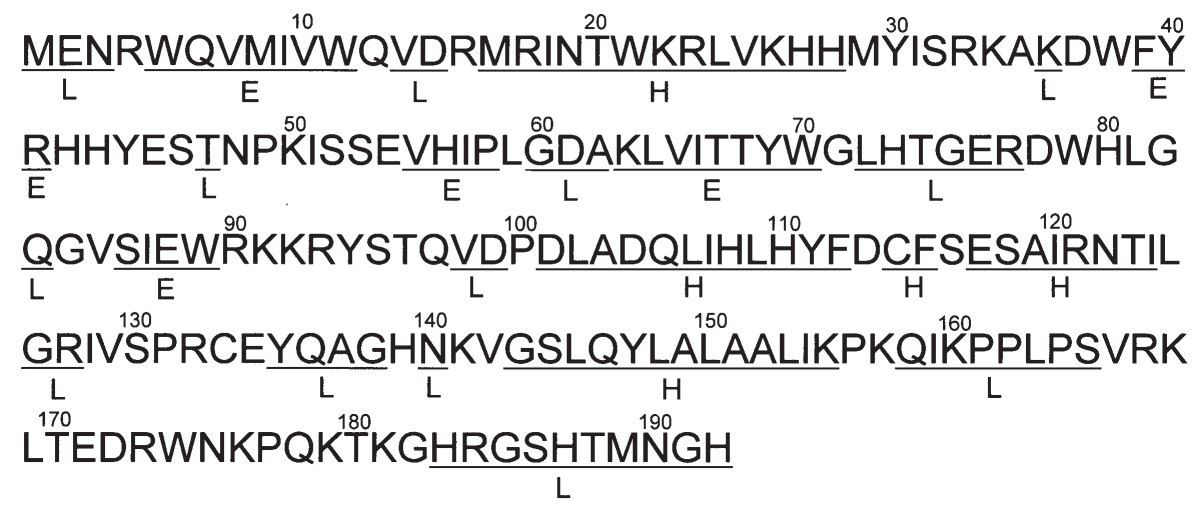

B

$\begin{array}{rlc}2226343650639192141155157160168176179181 & \text { level } & \text { infectivity } \\ \text { WT KKKKKKKKKKKKKKK } & \text { Low } & + \\ \text { K22/16R RRRRRRRRRRRRRRRR } & \text { High } & - \\ \text { K22/6R RRRRRRKKKKKKKKKK } & \text { Low } & - \\ \text { K91/10R KKKKKKRRRRRRRRRR } & \text { Low } & +\end{array}$

Figure 1. The lysine-arginine alterations in the first group of Vif mutants. Structure of the Vif of HIV-1 NL432 clone by the PredictProtein (http://www. predictprotein.org/) (A) and the alterations in this Vif (the first group mutants) (B) are indicated. L, E and H in (A) represent the loop, $\beta$-strand and $\alpha$-helix structures, respectively. Data on the expression level in cells of the mutated Vif proteins and the infectivity for H9 cells of the mutants are also shown in (B) (see Figure 2).

Reverse transcription (RT) assay. Virus production in the culture supernatants of transfected H9 cells was monitored by RT assay as previously described (27).

Western immunoblot analysis. Transfected 293T cells were collected and solubilized by dissolving in PBS-Laemmli's sample buffer (1:1) for SDS-PAGE as previously described (20). Samples resolved by the SDS-PAGE were then electrophoretically transferred to polyvinylidene fluoride membranes (Immobilon-P, Millipore, Bedford, MA, USA). The membranes were treated with anti-FLAG antibody (Ab) (ANTI-FLAG M2 Monoclonal Ab, Sigma-Aldrich, St. Louis, MO, USA) and visualized using the ECL plus Western blot detection system (Amersham Biosciences, Buckinghamshire, UK).

Pulse/chase analysis. Transfected 293T cells were pulselabelled with ${ }^{35} \mathrm{~S}$, and chased as previously described (20). Cells harvested were lysed with the CHAPS/DOC buffer as described previously (20), and the cell lysates were precipitated with a Vif-specific polyclonal antibody Vif93 (28). Wt and mutant Vif proteins were identified by SDS-PAGE followed by fluorography as previously described (20).

DNA constructs for infection experiments. An infectious proviral clone of HIV-1 designated pNL432 (26) was used as wt clone for infection experiments. Appropriate fragments of pNL432 were subcloned into pBluescript SK(+) (Stratagene, La Jolla, CA, USA) and mutations were introduced. The alterations of lysines into arginines were performed by the QuikChange site-directed mutagenesis kit (Stratagene). The mutated fragments were cloned back to wt to construct pNLK22/6R, pNL-K91/10R, pNL-K22/2R, pNL-K34/2R, pNLK50R, pNL-K63R, pNL-K22R and pNL-K26R. To make
pNL-K22/16R, appropriate fragments of pNL-K22/6R and pNL-K91/10R were used. Clone pNL-K34/14R was constructed from the pNL-K22/16R similarly as above. As a negative control, pNL-Nd (29) carrying a frame-shift mutation in vif was used.

DNA constructs for Western immunoblot and pulse/chase analyses. The pNL-A1S (21) was used to construct expression vectors for Western blot and pulse/chase analyses. To generate pNL-ASCF, a ClaI site and the FLAG sequence (in this order) were introduced just upstream of the stop codon of vif in pNL-A1S (21) by the QuikChange site-directed mutagenesis kit. The vif sequences of pNL432 and its mutants, pNL-K22/16R, pNL-K22/6R, pNL-K91/10R and pNL-K34/ $14 \mathrm{R}$, were amplified by polymerase chain reaction (PCR) with SmaI at 5' and ClaI at 3' ends, respectively. The SmaI-ClaI fragment from the pNL-ASCF was replaced with these PCRamplified sequences to construct pNL-ASCF-fWT, pNLASCF-fK22/16R, pNL-ASCF-fK22/6R, pNL-ASCF-fK91/ 10R and pNL-ASCF-fK34/14R. An appropriate fragment of pNL-ASCF-fK22/16R was subcloned into pBluescript SK(+), and mutations were introduced by the QuikChange sitedirected mutagenesis kit. The mutated fragments were cloned back to pNL-ASCF-fK22/16R to construct pNL-ASCF-K22/ 2R/50/12R, pNL-ASCF-K22/6R/141/8R, pNL-ASCF-K22/ 9R/160/5R and pNL-ASCF-K22/14R. As an expression vector for luciferase, pGL3-Control Vector (Promega, Madison, WI, USA) was used.

\section{Results}

Expression and infectivity of various HIV-1 Vif mutants. We examined the importance of lysines present in Vif for its 
A

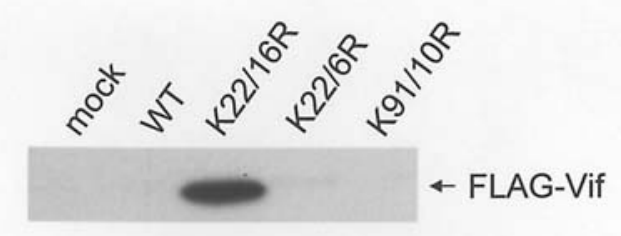

B

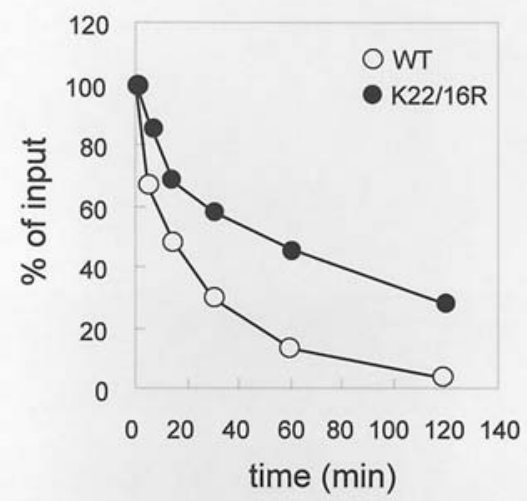

C

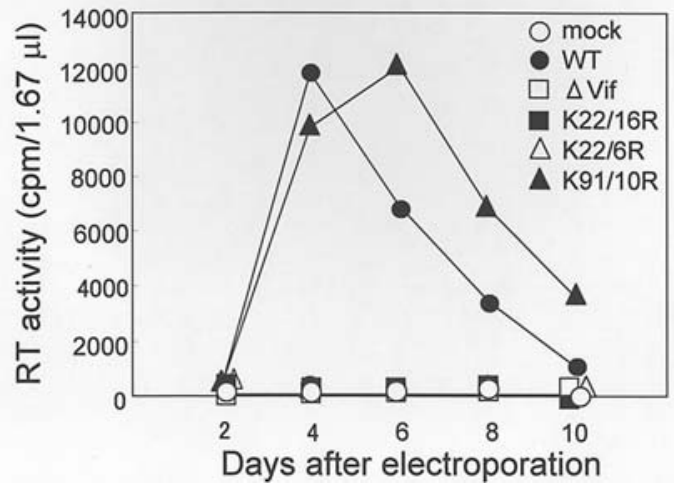

Figure 2. Characteristics of the first group of Vif mutants. (A) Expression level in cells of wt and mutant clones. Co-transfected 293T cells with $7.5 \mu \mathrm{g}$ of the subgenomic viral clones derived from pNL-ASCF-fWT and $2.5 \mu \mathrm{g}$ of an expression vector for luciferase, at 48-h post-transfection, were harvested for Western blot analysis and luciferase assay. The loading amount in each lane was normalized by the luciferase activity. mock, pUC19; WT, pNLASCF-fWT. (B) Degradation kinetics in $293 \mathrm{~T}$ cells of the K22/16R mutant. Transfected $293 \mathrm{~T}$ cells with $5 \mu \mathrm{g}$ of the subgenomic clones, and at $24-\mathrm{h}$ post-transfection, were collected, ${ }^{35} \mathrm{~S}$-labeled, and chased for $\leq 120 \mathrm{~min}$ for immunoprecipitation analysis. WT, pNL-ASCF-fWT. (C) Growth kinetics in non-permissive cells of wt and mutant clones. H9 cells were transfected with $10 \mu \mathrm{g}$ of the full-length viral clones, and virus production in the culture supernatants was monitored by RT assay. mock, pUC19; WT, pNL432; $\Delta$ Vif, a frame-shift mutant pNL-Nd.

expression and viral infectivity. As the first group mutants for this study, clones K22/16, K22/6R and K91/10R, which carry all 16, $\mathrm{N}$-terminal 6 and $\mathrm{C}$-terminal 10 lysine-ariginine exchanges, respectively, were constructed and characterized (Fig. 1). For a quantitative comparison, their expression to a high level was achieved by the subgenomic-type clone (21) and analyzed by Western immunoblotting using anti-FLAG antibody. As shown in Fig. 2A, the mutant K22/16R produced its Vif at an extremely high level relative to those of wt, K22/ 6R and K91/10R. We determined the stability of the K22/16R
A

$22 \quad 26 \quad 34 \quad 36 \quad 5063 \quad 91 \quad 92141155157160168176179181$ WT KKKKKKKKKKKKKKKK + K22/2R R R K K K K K K K K K K K K K K34/2R K K R R K K K K K K K K K K K + K50R K K K KRKKKKKKKKKKK + K63R K K K K K R K K K K K K K K K K + K22R R K K K K K K K K K K K K K K K + K26R KR K K K K K K K K K K K K K K + K34/14R K K R R R R R R R R R R R +

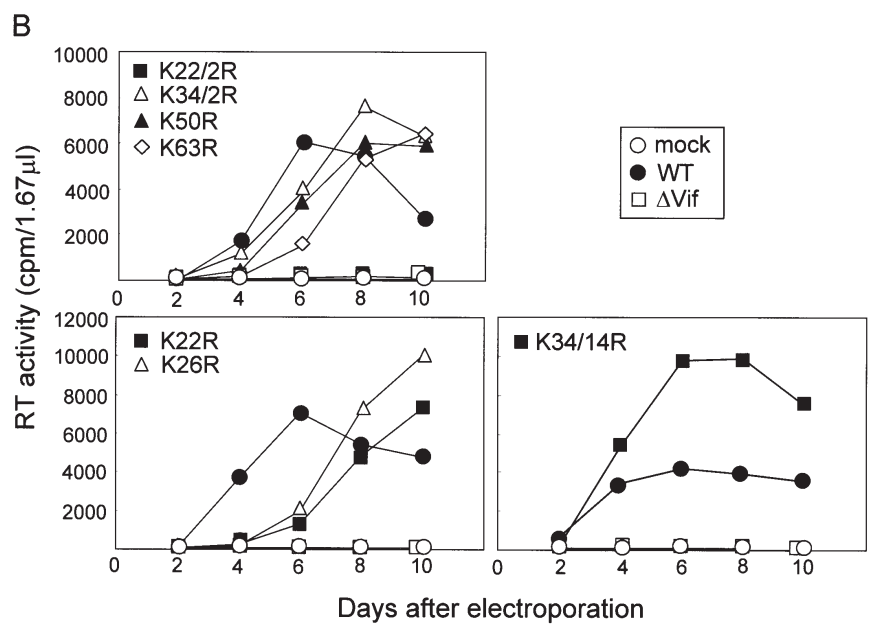

Figure 3. Identification of the lysine residue in Vif critical for viral infectivity. Location of the lysine to arginine alterations in the Vif of the mutants (A) and their growth kinetics in H9 cells (B) are shown. For determination of viral infectivity in $\mathrm{H} 9$ cells, cells were transfected with various full-length clones and monitored for virus production as above. Data in (B) are summarized in (A) as indicated. mock, pUC19; WT, pNL432; $\Delta$ Vif, a frame-shift mutant $\mathrm{pNL}-\mathrm{Nd}$.

Vif by the pulse/chase experiment as previously described (20). As shown in Fig. 2B, in a good agreement with the steadystate expression level, K22/16R was much more stable than wt. We next examined the infectivity of these mutants for non-permissive cells. H9 cells were electroporated with the full-length version of the mutant clones, and virus growth was monitored by RT assay (30). As shown in Fig. 2C, only the mutant K91/10R among the three mutants, which express a high or negligible level of Vif in cells (Fig. 2A), grew fairly well.

In total, our results described herein indicated that the lysines present in Vif were important for the stable expression of Vif, and that there is no clear negative or positive corelationship between the expression level of Vif and infectivity of lysine-ariginine mutants.

Lysine residues in Vif important for viral infectivity. To determine the lysine residue in Vif crucial for viral infectivity, we constructed another set of proviral mutant clones. One or two lysines located at the N-terminal region of Vif were changed to the arginine residue (K22/2R, K34/2R, K50R and K63R in Fig. 3A), and the resultant clones were examined for their growth kinetics in H9 cells as above. As shown in Fig. 3B, only the K22/2R were not infectious. Therefore, we 
A

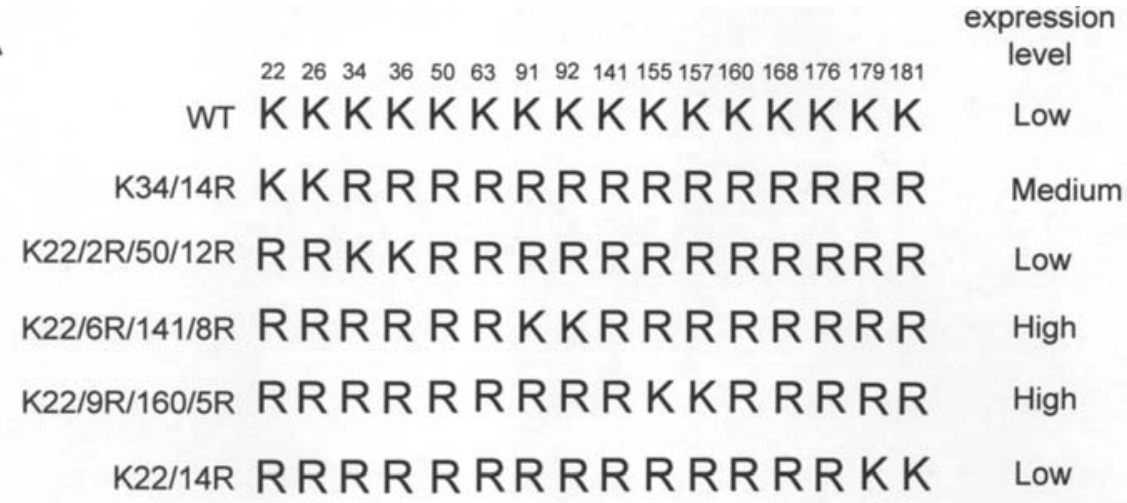

B

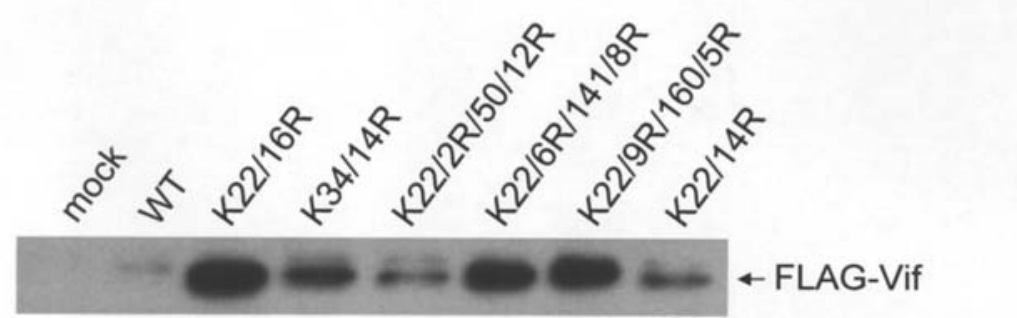

Figure 4. Identification of the lysine residue in Vif critical for the wt expression level within cells. Location of the lysine to arginine alterations in the Vif of the mutants (A) and their expression in the transfected 293T cells (B) are shown. For monitoring the expression level in 293T cells of the Vif, cells were transfected with various subgenomic clones and analyzed for their expression as above. Data in (B) are summarized in (A) as indicated. mock, pUC19; WT, pNL-ASCF-fWT.

constructed K22R, K26R and K34/14R (Fig. 3A) to determine whether one of the $\mathrm{K}^{22}$ and $\mathrm{K}^{26}$ or both are essential for viral infectivity, and whether the lysines other than $\mathrm{K}^{22}$ and $\mathrm{K}^{26}$ are critical for viral infectivity. The three additional mutants thus constructed were transfected into H9 cells, and their growth was examined. As shown in Fig. 3B, the three mutants grew to a comparable extent. All the mutants described above were confirmed to propagate in permissive M8166 cells (data not shown). Collectively, we concluded that either $\mathrm{K}^{22}$ or $\mathrm{K}^{26}$ of Vif is critical for the productive infection of HIV-1 NL432 in non-permissive cells.

Lysine residues in Vif important for the expression level of Vif. To determine the lysine residue in Vif crucial for the Vif expression, we constructed a series of double lysine-arginine mutants other than the K34/14R, based on the subgenomic clone (21) (Fig. 4A). The mutants were then analyzed for their Vif expression by Western immunoblotting as described above. When the mutant K34/14R, which is infectious for H9 cells (Fig. 3B), was monitored for its Vif expression, it displayed a medium expression level between wt and the stable and non-infectious mutant K22/16R (Fig. 4B). This observation again indicated the absence of a detectable close relationship between viral infectivity and the Vif expression level. The data on the other mutants in Fig. 4B clearly showed that the $\mathrm{K}^{34}$ and $\mathrm{K}^{36}$ or the $\mathrm{K}^{179}$ and $\mathrm{K}^{181}$ are responsible for the low expression level of Vif. While the mutants K22/6R/141/8R and K22/9R/160/5R produced a similarly high expression level to that of the K22/16R, the mutants K22/2R/50/12R and K22/14R expressed a low level of Vif quite similar to the wt clone. We also constructed a complete set of single lysine-arginine mutants through one by one alterations to evaluate the contribution of each lysine to the Vif expression. All the single mutants constructed were found to produce Vif, upon transfection, at a level higher than that of the mutants $\mathrm{K} 22 / 2 \mathrm{R} / 50 / 12 \mathrm{R}$ and $\mathrm{K} 22 / 14 \mathrm{R}$ (data not shown). These results strongly suggested that each lysine residue in Vif is less important than the combination of $\mathrm{K}^{34}$ and $\mathrm{K}^{36}$ or of $\mathrm{K}^{179}$ and $\mathrm{K}^{181}$ for the wt expression level of Vif.

\section{Discussion}

In this study, we showed by a mutational analysis that the lysines in HIV-1 Vif are important for its steady-state expression in transfected $293 \mathrm{~T}$ cells, and that the two lysines in Vif ( $\mathrm{K}^{34}$ and $\mathrm{K}^{36}$ or $\mathrm{K}^{179}$ and $\mathrm{K}^{181}$ ) are nearly sufficient for the expression property of the Vif (Figs. 2 and 4). We also demonstrated that either $\mathrm{K}^{22}$ or $\mathrm{K}^{26}$ in Vif is critical for the replication of HIV-1 in H9 cells (Figs. 2 and 3). Thus, we did not find any clear co-relationship between the expression level in cells of Vif and viral infectivity for the non-permissive cells.

Together with the results previously published $(20,22)$, it was reasonable to assume that the $\mathrm{K}^{34}$ and $\mathrm{K}^{36}$ or $\mathrm{K}^{179}$ and $\mathrm{K}^{181}$ are important to maintain the low appropriate expression level of Vif by the proteasome-degradation, and thereby enable the virus to grow in non-permissive cells. However, our data herein on the mutants demonstrating that either $\mathrm{K}^{22}$ or $\mathrm{K}^{26}$ is critical for viral infectivity do not support this prediction, and instead, the mechanism(s) and molecule(s) other than the proteasomedegradation and its associated factors which confer the infectivity on the virus should be considered. They would include 1) the structure of the lysine itself, 2) covalent modification(s) of the lysine and 3) interaction of the lysine 
with some unknown factor(s). Further study is required to clarify the molecular basis for our observations on the Vif mutants.

\section{Acknowledgements}

We thank Ms. Kazuko Yoshida for editorial assistance. This work was supported by a Grant-in-Aid for Scientific Research (B) (18390140) and a Grant-in-Aid for Scientific Research (C) (15590420) from the Japan Society for the Promotion of Science, and a Health Sciences Research Grant (Research on HIV/AIDS 16150301) from the Ministry of Health, Labor and Welfare of Japan. B.K. is supported by the Royal Thai Government Scholarship 2003 (Commission on Higher Education).

\section{References}

1. Miller RJ, Cairns JS, Bridges S and Sarver N: Human immunodeficiency virus and AIDS: insights from animal lentiviruses. J Virol 74: 7187-7195, 2000.

2. Harada S, Koyanagi Y and Yamamoto N: Infection of HTLV-III/ LAV in HTLV-I-carrying cells MT-2 and MT-4 and application in a plaque assay. Science 229: 563-566, 1985.

3. Shibata R, Kawamura M, Sakai H, Hayami M, Ishimoto A and Adachi A: Generation of a chimeric human and simian immunodeficiency virus infectious to monkey peripheral blood mononuclear cells. J Virol 65: 3514-3520, 1991

4. Mann DL, O'Brien SJ, Gilbert DA, Reid Y, Popovic M, Read-Connole E and Gallo RC: Origin of the HIV-susceptible human $\mathrm{CD}^{+}$cell line H9. AIDS Res Hum Retrov 5: 253-255, 1989.

5. Gabuzda DH, Lawrence K, Langhoff E, Terwilliger E, Dorfman T, Haseltine WA and Sodroski J: Role of vif in replication of human immunodeficiency virus type 1 in $\mathrm{CD}^{+} \mathrm{T}$ lymphocytes. J Virol 66: 6489-6495, 1992.

6. Sakai H, Shibata R, Sakuragi JI, Sakuragi S, Kawamura M and Adachi A: Cell-dependent requirement of human immunodeficiency virus type 1 Vif protein for maturation of virus particles. J Virol 67: 1663-1666, 1993.

7. von Schwedler U, Song J, Alken C and Trono D: vif is crucial for human immunodeficiency virus type 1 proviral DNA synthesis in infected cells. J Virol 67: 4945-4955, 1993.

8. Borman AM, Quillent C, Charneau P, Dauguet C and Clavel F: Human immunodeficiency virus type 1 Vif-mutant particles from restrictive cells: role of Vif in correct particle assembly and infectivity. J Virol 69: 2058-2067, 1995.

9. Simon JHM and Malim MH: The human immunodeficiency virus type 1 Vif protein modulates the postpenetration stability of viral nucleoprotein complexes. J Virol 70: 5297-5305, 1996.

10. Sheehy AM, Gaddis NC, Choi JD and Malim MH: Isolation of a human gene that inhibits HIV-1 infection and is suppressed by the viral Vif protein. Nature 418: 645-650, 2002.

11. Conticello SG, Harris RS and Neuberger MS: The Vif protein of HIV triggers degradation of the human antiretroviral DNA deaminase APOBEC3G. Curr Biol 13: 2009-2013, 2003.

12. Kao S, Khan MA, Miyagi E, Plishka R, Buckler-White A and Strebel K: The human immunodeficiency virus type 1 Vif protein reduces intracellular expression and inhibits packaging of APOBEC3G (CEM15), a cellular inhibitor of virus infectivity. J Virol 77: 11398-11407, 2003

13. Mariani R, Chen D, Schrofelbauer B, Navarro F, Konig R, Bollman B, Munk C, Nymark-McMahon and Landau NR: Species-specific exclusion of APOBEC3G from HIV-1 virions by Vif. Cell 114: 21-31, 2003.
14. Marin M, Rose KM, Kozak SL and Kabat D: HIV-1 Vif protein binds the editing enzyme APOBEC $3 \mathrm{G}$ and induces its degradation. Nat Med 9: 1398-1403, 2003.

15. Sheehy AM, Gaddis NC and Malim MH: The antiretroviral enzyme APOBEC $3 \mathrm{G}$ is degraded by the proteasome in response to HIV-1 Vif. Nat Med 9: 1404-1407, 2003.

16. Stopak K, de Noronha C, Yonemoto W and Greene WC: HIV-1 Vif blocks the antiviral activity of APOBEC 3G by impairing both its translation and intracellular stability. Mol Cell 12: 591-601, 2003.

17. Yu X, Yu Y, Liu B, Luo K, Kong W, Mao P and Yu XF: Induction of APOBEC $3 \mathrm{G}$ ubiquitination and degradation by an HIV-1 Vif-Cul5-SCF complex. Science 302: 1056-1060, 2003.

18. Kao S, Miyagi E, Khan MA, Takeuchi H, Opi S, Goila-Gaur R and Strebel K: Production of infectious human immunodeficiency virus type 1 does not require depletion of APOBEC $3 \mathrm{G}$ from virus-producing cells. Retrovirology 1: 27, 2004.

19. Mehle A, Strack B, Ancuta P, Zhang C, McPike M and Gabuzuda D: Vif overcomes the innate antiviral activity of APOBEC $3 \mathrm{G}$ by promoting its degradation in the ubiquitinproteasome pathway. J Biol Chem 279: 7792-7798, 2004.

20. Fujita M, Akari H, Sakurai A, Yoshida A, Chiba T, Tanaka K, Strebel K and Adachi A: Expression of HIV-1 accessory protein Vif is controlled uniquely to be low and optimal by proteasome degradation. Microbes Infect 6: 791-798, 2004.

21. Wang H, Sakurai A, Khamsri B, Uchiyama T, Gu H, Adachi A and Fujita M: Unique characteristics of HIV-1 Vif expression. Microbes Infect 7: 385-390, 2005.

22. Akari H, Fujita M, Kao S, Khan MA, Shehu-Xhilaga M, Adachi A and Strebel K: High level expression of human immunodeficiency virus type-1 Vif inhibits viral infectivity by modulating proteolytic processing of the Gag precursor at the $\mathrm{p} 2 / \mathrm{NC}$ processing site. J Biol Chem 279: 12355-12362, 2004.

23. Strebel K, Daugherty D, Clouse K, Cohen D, Folks T and Martin MA: The HIV 'A' (sor) gene product is essential for virus infectivity. Nature 328: 728-730, 1987.

24. Pickart CM: Back to the future with ubiquitin. Cell 116: 181-190, 2004.

25. Lebkowski JS, Clancy S and Calos MP: Simian virus 40 replication in adenovirus-transformed human cells antagonizes gene expression. Nature 317: 169-171, 1985.

26. Adachi A, Gendelman HE, Koenig S, Folks T, Willey R, Rabson A and Martin MA: Production of acquired immunodeficiency syndrome-associated retrovirus in human and nonhuman cells transfected with an infectious molecular clone. J Virol 59: 284-291, 1986.

27. Willey RL, Smith DH, Lasky LA, Theodore TS, Earl PL, Moss B, Capon DJ and Martin MA: In vitro mutagenesis identifies a region within the envelope gene of the human immunodeficiency virus that is critical for infectivity. J Virol 62: 139-147, 1998.

28. Karczewski MK and Strebel K: Cytoskeleton association and virion incorporation of the human immunodeficiency virus type 1 Vif protein. J Virol 70: 494-507, 1996.

29. Adachi A, Ono N, Sakai H, Ogawa K, Shibata R, Kiyomatsu T, Masuike $\mathrm{H}$ and Ueda S: Generation and characterization of the human immunodeficiency virus type 1 mutants. Arch Virol 117: $45-58,1991$.

30. Fujita M, Yoshida A, Miyaura M, Sakurai A, Akari H, Koyama AH and Adachi A: Cyclophilin A-independent replication of a human immunodeficiency virus type 1 isolate carrying a small portion of the simian immunodeficiency virus SIVMAC gag capsid region. J Virol 75: 10527-10531, 2001. 